\title{
The development of e-MERLIN
}

DOI:

10.1093/astrogeo/atw101

\section{Document Version}

Final published version

Link to publication record in Manchester Research Explorer

\section{Citation for published version (APA):}

Garrington, S., \& Beswick, R. (2016). The development of e-MERLIN. Astronomy and Geophysics, 57(3), 3.283.30. https://doi.org/10.1093/astrogeo/atw101

\section{Published in:}

Astronomy and Geophysics

\section{Citing this paper}

Please note that where the full-text provided on Manchester Research Explorer is the Author Accepted Manuscript or Proof version this may differ from the final Published version. If citing, it is advised that you check and use the publisher's definitive version.

\section{General rights}

Copyright and moral rights for the publications made accessible in the Research Explorer are retained by the authors and/or other copyright owners and it is a condition of accessing publications that users recognise and abide by the legal requirements associated with these rights.

\section{Takedown policy}

If you believe that this document breaches copyright please refer to the University of Manchester's Takedown Procedures [http://man.ac.uk/04Y6Bo] or contact uml.scholarlycommunications@manchester.ac.uk providing relevant details, so we can investigate your claim.

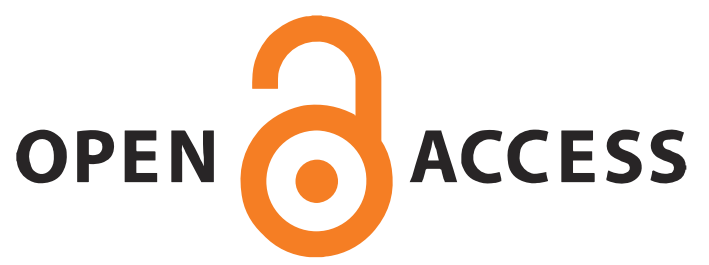




\section{The development of e-MERLIN}

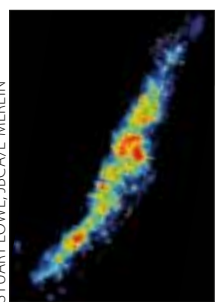

Deep in the heart of the Cheshire countryside lies Jodrell Bank Observatory, home to the UK's national facility for high-resolution radio astronomy: e-MERLIN, the enhanced Multi-Element Remote-Linked Interferometer Network. In this issue of $A \& G$, the e-MERLIN team highlights some of its cutting-edge experiments, probing fundamental physical processes in our own galaxy, and reaching further out to higher redshift where radio astronomy can investigate cosmic history and evolution. In this first article, Simon Garrington and Rob Beswick explain how e-MERLIN came to be.

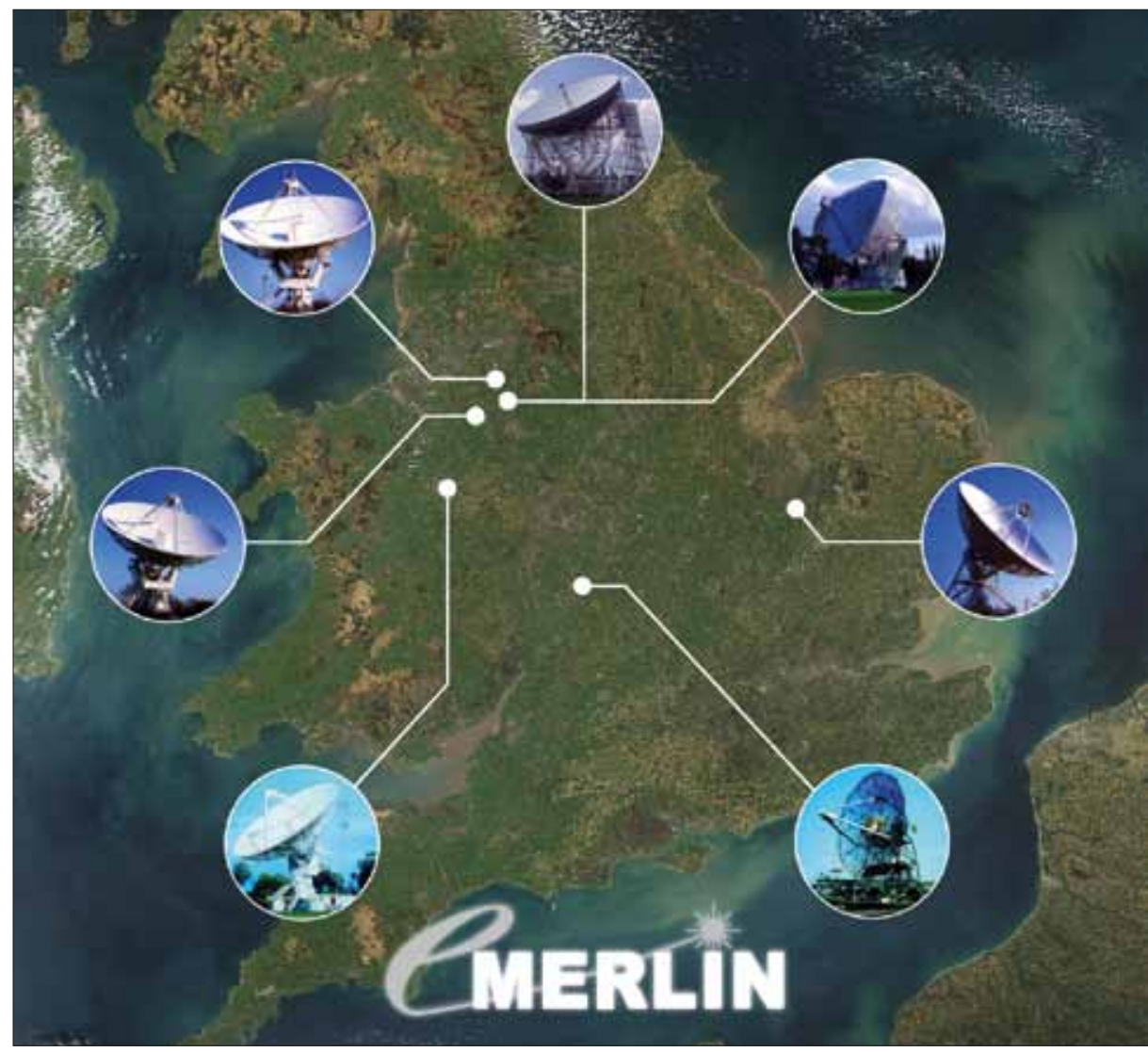

1 The locations of the e-MERLIN telescopes. Clockwise from top: Jodrell Bank Lovell, Jodrell Bank Mk2, Cambridge, Defford, Knockin, Darnhall and Pickmere. Linking them boosts the resolution, and optical fibre connections to increase the bandwidth enhance the sensitivity. (S Lowe/JBCA, Univ. Manchester)

T he foundations of e-MERLIN were laid in the 1940s, after the second world war. At this time, the scientists who developed war-time radar-including Bernard Lovell and Martin Ryle - returned to their laboratories and began experiments in what would become the new science of radio astronomy. It was an intriguing field: only a few discrete radio sources were known and their nature was a mystery. Within a few years, Francis Graham-Smith at Cambridge had localized one of the brightest, Cygnus A, allowing Walter Baade and Rudolph Minkowski at Mount Palomar to secure an optical identification, showing that the emission appeared to come from a pair of colliding galaxies. To better localize the radio emission required much greater resolution, and hence a much greater separation between antennas. Roger Jennison and his student Mrinal das Gupta at Jodrell Bank achieved this by connecting pairs of antennas with a radio link, rather than by cable. As they extended the separation of their antennas to $5 \mathrm{~km}$, the structure of Cygnus A emerged: two huge lobes of radio emission beyond the visible galaxy.

Experiments in interferometry continued through the turbulent period of the construction of the Lovell Telescope at Jodrell Bank in the 1950s, when Bernard Lovell balanced research and national security goals with an ever-increasing mound of debt (Lovell 2007). A small team led by Henry Palmer extended the antenna separation to $20 \mathrm{~km}$ and discovered that several radio objects were much smaller - less than about 10 arcsec - than had been assumed. It was possible that they were radio stars rather than galaxies. Using a newly installed $750 \mathrm{ft}$ TV mast, the team was able to achieve a baseline of $115 \mathrm{~km}$, long enough to match the 1 arcsec resolution of optical telescopes. Astronomers at Caltech pursued the most 


\section{Legacy e-MERLIN programmes}

\begin{tabular}{|c|c|c|}
\hline project & principal investigator & $\begin{array}{c}\text { allocation } \\
\text { (hrs) }\end{array}$ \\
\hline $\begin{array}{l}\text { Astrophysics of Galaxy Transformation and Evolution } \\
\text { (AGATE) }\end{array}$ & $\begin{array}{l}\text { C Simpson (Cape Town), } \\
\text { I Smail (Durham) }\end{array}$ & 330 \\
\hline $\begin{array}{l}\text { The e-MERLIN Cyg OB2 Radio Survey: Massive and } \\
\text { Young Stars in the Galaxy (COBRaS) }\end{array}$ & R Prinja (UCL) & 294 \\
\hline e-MERLIN Galaxy Evolution Survey (eMERGE) & $\begin{array}{l}\text { T Muxlow (Manchester), } \\
\text { I Smail (Durham), } \\
\text { I McHardy (Southampton) }\end{array}$ & 918 \\
\hline e-MERLIN Pulsar Interferometry Project (e-PI) & $\begin{array}{l}\text { W Vlemmings (Onsala, SE), } \\
\text { B Stappers (Manchester) }\end{array}$ & 160 \\
\hline Feedback Processes in Massive Star Formation & $\begin{array}{l}\text { M Hoare (Leeds), } \\
\text { W Vlemmings (Onsala SE) }\end{array}$ & 450 \\
\hline $\begin{array}{l}\text { Gravitational Lensing and Galaxy Evolution with } \\
\text { e-MERLIN }\end{array}$ & $\begin{array}{l}\text { N Jackson (Manchester), } \\
\text { S Sargent (OU) }\end{array}$ & 228 \\
\hline $\begin{array}{l}\text { Legacy e-MERLIN Multi-Band Imaging of Nearby } \\
\text { Galaxies (LeMMINGs) }\end{array}$ & $\begin{array}{l}\text { R Beswick (Manchester), } \\
\text { I McHardy (Southampton) }\end{array}$ & 810 \\
\hline Luminous Infra-red Galaxy Inventory (LIRGI) & $\begin{array}{l}\text { J Conway (Onsala, SE), } \\
\text { M Pérez-Torres (IAA, ES) }\end{array}$ & 353 \\
\hline $\begin{array}{l}\text { Morphology and Time Evolution of Thermal Jets } \\
\text { Associated with Low Mass Young Stars }\end{array}$ & $\begin{array}{l}\text { L Rodriguez (UNAM, } \\
\text { Mexico) }\end{array}$ & 180 \\
\hline $\begin{array}{l}\text { Planet Earth Building Blocks - a Legacy e-MERLIN } \\
\text { Survey (PEBBLES) }\end{array}$ & J Greaves (Cardiff) & 72 \\
\hline Resolving Key Questions in Extragalactic Jet Physics & $\begin{array}{l}\text { R Laing (ESO), } \\
\text { M Hardcastle (Herts) }\end{array}$ & 375 \\
\hline $\begin{array}{l}\text { Super-CLASS: the Super-CLuster Assisted Shear Survey } \\
\text { - a weak lensing deep field survey using e-MERLIN }\end{array}$ & R Battye (Manchester) & 832 \\
\hline
\end{tabular}

compact objects found by the Jodrell team and matched the radio source 3C48 with what appeared to be a radio star with peculiar spectral lines. With the identification of 3C273 at the Parkes radio telescope in Australia in 1962-63, the significance of these compact objects became clear: they were a new class of body called quasistellar objects or quasars.

\section{Matching the Hubble}

The long-baseline interferometers developed at Jodrell Bank were ideally suited to studying these new objects and by 1980 a permanent network of dishes radiolinked to Jodrell Bank, was established: MERLIN, the Multi-Element Radio-Linked Interferometer Network (Thomasson 1986). Extended in 1990 to include a new $32 \mathrm{~m}$ dish near Cambridge, the $217 \mathrm{~km}$ span of MERLIN (figure 1), operating at centimetre wavelengths, could match the angular resolution of the Hubble Space Telescope in the optical band. The ability to compare radio and optical emission at subarcsecond resolution brought a unique insight across fields from the formation and evolution of stars to the nature of the energetic outflows from quasars and radio galaxies.

After the publication of the first Hubble Deep Field images in 1996, MERLIN was among the many telescopes to make deep observations of the same field. Radio observations provide a measure of starforming activity free from obscuration by dust, and can therefore make a major contribution to our understanding of the history of star formation in the universe. Despite the heroic efforts from observers (18 days of observation and years of data processing), MERLIN detected only a small fraction of the thousands of galaxies seen in the deepest optical images. MERLIN's unique ability to match the resolution of Hubble came at a price: the long radio-linked baselines limited bandwidth, ........... and hence sensitivity. telescopes with optical MERLIN could detect and fibres, bandwidth was image the radio emission increased 100-fold" from star formation and distant active galactic nuclei led to an ambitious project to realize the full potential of the network. By connecting the existing telescopes with optical fibres, not radio, the bandwidth could be increased at least 100 -fold. Together with improved receivers and a new surface on the Lovell Telescope, the sensitivity of this new instrument, e-MERLIN, would be 30 times better (Spencer 2009).

Building the optical fibre network was one of the main challenges. The network, among others, needed a traffic rate of at
What can radio astronomy reveal?

Radio waves at frequencies between about $10 \mathrm{MHz}$ (wavelength $30 \mathrm{~m}$ ) and $1 \mathrm{THz}(0.3 \mathrm{~m})$ are used for ground-based astronomy. Just as for visible light, radio emission can be collected as a broadband signal, spread across many frequencies, or in the form of spectral features that allow determination of the chemical composition and relative velocity of an object, for example.

Most radio emission can be identified as arising from either thermal or nonthermal processes. The cosmic microwave background is a well-known source of radio emission, with a thermal black-body spectrum peaking at a wavelength of $\sim 1 \mathrm{~mm}$. Thermal emission in the radio part of the spectrum is also generated by warm gas, often in regions where stars form. Dust grains render these regions opaque in many parts of the spectrum, but as particle size is smaller than radio wavelengths, the dusty interstellar medium (ISM) is nearly transparent to radio telescopes. This allows us to peer through the dusty disc of the Milky Way to find the compact radio source Sgr A* the supermassive black hole at the centre of our galaxy. Non-thermal synchrotron emission identifies where electrons have been accelerated, for example by supernova shocks, or in lobes of charged particles accelerated by vast high-energy jets from massive black holes in active galactic nuclei of radio galaxies.

Specific target frequencies allow radio astronomers to address key astrophysical processes. For example, tracking the $21 \mathrm{~cm}$ line from atomic hydrogen allows mapping of this species, which makes up three-quarters of the visible universe. This technique can probe environments such as giant molecular clouds, where stars are forming, stellar and planetary discs and the dusty regions of galaxies, including the Milky Way.

At millimetre and submillimetre wavelengths, especially, there are emission lines from molecular species such as water, ammonia and organic molecules. Radio data are important for understanding the origin of life in the universe and for examining the composition of the growing number of planets around other stars.

The hydroxyl ion $\left(\mathrm{OH}^{-}\right)$, among others, produces unusually narrow and polarized emission lines as a result of a natural amplification process called a maser (analogous to a laser). Maser emission is useful because it is amplified; the specific conditions needed for amplification also give astronomers information about the density and velocity of the species in the source region, its temperature and the existence of magnetic fields. 


\section{Final parameters of e-MERLIN}

\begin{tabular}{|c|c|c|c|c|}
\hline & $\begin{array}{l}1.5 \mathrm{GHz} \\
\text { (L band) }\end{array}$ & $\begin{array}{c}5 \mathrm{GHz} \\
(\mathrm{C} \text { band })\end{array}$ & $\begin{array}{c}22 \mathrm{GHz} \\
\text { (K band) }\end{array}$ & notes \\
\hline resolution (milliarcseconds) & 150 & 40 & 12 & uniform weighting at central frequency \\
\hline field of view (arcmin) & 30 & 7 & 2.0 & FWHM of $25 \mathrm{~m}$ dishes; reduced when Lovell Telescope included at 1.5 or $5 \mathrm{GHz}$ \\
\hline frequency range $(\mathrm{GHz})$ & $1.3-1.7$ & $4-8$ & $22-24$ & \\
\hline bandwidth (GHz) & 0.4 & 2 & 2 & $\begin{array}{l}\text { maximum bandwidth per polarization; at } \mathrm{C} \text { or } \mathrm{K} \text { band, } 4 \mathrm{GHz} \text { bandwidth is } \\
\text { possible using a single polarization }\end{array}$ \\
\hline sensitivity $(\mu \mathrm{Jy} / \mathrm{bm})$ in full imaging run & $5-6$ & $1.8-2.3$ & $\sim 15$ & \multirow{2}{*}{$\begin{array}{l}\text { final performance will depend on usable bandwidth, final receiver } \\
\text { optimization, Lovell performance; figures are for e-MERLIN with Lovell }\end{array}$} \\
\hline surface brightness sensitivity (K) & 190 & $\sim 70$ & $\sim 530$ & \\
\hline ICRF astrometric performance (mas) & 2 & $\sim 1$ & $\sim 2$ & $\begin{array}{l}\text { with respect to the ICRF (typical } 3^{\circ} \text { target-calibrator separation using the } \\
\text { VLBA Calibrator Survey) }\end{array}$ \\
\hline astrometric repeatability (mas) & $\sim 0.5$ & $\sim 0.2$ & $\sim 1$ & $\begin{array}{l}\text { day-to-day repeatability using surveyed or in-beam sources, and assuming } \\
\text { full imaging run }\end{array}$ \\
\hline amplitude calibration & 2 & 1 & 10 & targets for day-to-day repeatability \\
\hline
\end{tabular}

The Lovell Telescope may be included in e-MERLIN when observing at 1.5 and $5 \mathrm{GHz}$. Its inclusion increases the sensitivity by a factor of between 2 and 3 and reduces the field of view to approximately $20 \mathrm{arcmin}$ (at $1.4 \mathrm{GHz}$ ), depending on the data-weighting scheme adopted.

least $150 \mathrm{~Gb} \mathrm{~s}^{-1}$; at the time the tenders were issued, the total UK internet traffic was less than one tenth of that. The solution involved several telecoms providers and approximately $100 \mathrm{~km}$ of new fibre to reach the remote rural locations of the telescopes. Commercially available systems were not good enough, so e-MERLIN uses a modified version of equipment designed by the US National Radio Astronomy Observatory and Jodrell Bank Observatory for the Jansky Very Large Array and the Atacama Large Millimetre Array.

To achieve the increased bandwidth, the e-MERLIN team had to replace almost every component of the array, except for the main steel structures of the telescopes. Complete new $4-8 \mathrm{GHz}$ receivers were built, including new feeds, amplifiers and polarizers, all of which were designed at JBO. To increase observing flexibility, a new lens system was installed at three telescopes, together with new broadband feeds for observations at 1.3-1.8 GHz. All the telescope drive systems and control computers are being upgraded, the analogue and digital signal processing systems have been replaced, and a powerful new highly flexible correlator was installed, designed and built by Dominion Radio Astronomy Observatory in Penticton, Canada.

One of the key innovations that made MERLIN feasible was the microwave frequency transfer system, which delivered a coherent frequency standard to each telescope from a hydrogen maser clock at Jodrell Bank. For e-MERLIN this was re-worked to modulate an additional pair of optical channels on the same fibres that transport the data from the telescopes. This system transfers the clock signal with an accuracy of $\sim 1$ ps across the entire network.

Such a whole-scale upgrade was a challenge. The first fringes were obtained in 2009, the first test images with the full network at the end of 2010, and routine operations started in 2012.

e-MERLIN is now mid-way through a major legacy programme, devised to give significant observing time to major science projects early in the network's operation.

....... The overall goal is to address erful combination of arrays fundamental questions in astronomy and astrophysics, while also producing a substantial data legacy for the astronomical community.

The e-MERLIN Legacy Steering Group selected 12 large projects, probing astrophysical phenomena from planet and star formation to cosmology, shown in table 1. Observations for the e-MERLIN legacy programme are more than $50 \%$ complete, with those requiring the broadband C-band capabilities awaiting completion of this system in autumn 2016. High-profile science results are already emerging.

\section{Open for research}

Beyond the e-MERLIN legacy programme, half of the working telescope time is open to proposals from researchers. These projects are fully supported, making the instrument accessible to scientists regardless of expertise. There are regular calls for proposals, which are assessed by the STFC Panel for the Allocation of Telescope Time.

Although e-MERLIN is now fully operational (see table 2 for the final parameters), technical development is continuing: improved 4-8GHz low-noise amplifiers are being installed to optimize performance with the new $2 \mathrm{GHz}$ bandwidth samplers, and new K-band $(18-26 \mathrm{GHz})$ receivers have been developed with new amplifiers, improving the system temperature by $30 \mathrm{~K}$.

Legacy project teams are also driving the development of e-MERLIN. For example, the COBRaS team is developing software for automated detection and removal of radio frequency interference; the e-MERGE team is demonstrating the ultimate performance of e-MERLIN at the L-band, and devising techniques for fast wide-field mapping; and the SuperCLASS team is investigating the wide-field polarization performance and developing sophisticated mosaicking techniques.

As we approach the era when the first phase of the Square Kilometre Array (SKA) becomes operational (see article on page 3.25 this issue), e-MERLIN will continue to play an important role. It is the only instrument with similar baseline lengths and frequency coverage to SKA-Mid, so it can guide related SKA projects, as well as providing a facility to train and develop the UK astronomical community. Working with the European VLBI Network, e-MERLIN can provide an instrument with baselines from $10 \mathrm{~km}$ to $10000 \mathrm{~km}$ and a total collecting area comparable to that of SKA1. This powerful combination of arrays will offer an important complement to SKA1 for sensitive highresolution imaging and surveys. $\bullet$

\section{AUTHORS}

Simon Garrington is associate director of Jodrell Bank Observatory at the University of Manchester, UK. Rob Beswick is e-MERLIN project scientist at the University of Manchester.

\section{ACKNOWLEDGMENTS}

e-MERLIN is a National Facility operated by the University of Manchester at Jodrell Bank Observatory on behalf of STFC.

\section{REFERENCES}

Lovell B 2007 Astron. \& Geophys. 485.21

Spencer RE 2009 Proc. 8th International e-VLBI Workshop 22-26 June 200929 http://pos.sissa.it/cgi-bin/reader/conf.cgi?confid=82 Thomasson P 1986 Quart. J. Royal Astron. Soc. 27413 